\title{
Resistance to antivascular endothelial growth factor treatment in age-related macular degeneration
}

This article was published in the following Dove Press journal:

Drug Design, Development and Therapy

14 June 2013

Number of times this article has been viewed

\author{
Paris Tranos' \\ Athanasios Vacalis' \\ Solon Asteriadis' \\ Stavrenia Koukoula' \\ Athanasios Vachtsevanos' \\ Georgia Perganta' \\ Ilias Georgalas ${ }^{2}$ \\ 'Retina Centre, Thessaloniki, Greece; \\ ${ }^{2}$ Department of Ophthalmology, \\ "G Gennimatas" Hospital of Athens, \\ University of Athens, Athens, Greece
}

Correspondence: llias Georgalas Department of Ophthalmology, University of Athens, 59 Chrysanthemon St, I5452 Athens, Greece

Tel +30 2l 07768000

Fax +30 21 07768000

Email igeorgalas@yahoo.com

\begin{abstract}
Age-related macular degeneration (AMD) is the main cause of visual impairment and blindness in people aged over 65 years in developed countries. Vascular endothelial growth factor (VEGF) is a positive regulator of angiogenesis and its proven role in the pathological neovascularization in wet AMD has provided evidence for the use of anti-VEGF agents as potential therapies. In this study, we review the literature for the possible causes of failure after treatment with anti-VEGF agents and attempt to propose an algorithm of suggestive actions to increase the chances of successful management of such difficult cases.
\end{abstract}

Keywords: antiVEGF, age related macular degeneration, treatment

\section{Introduction}

Age-related macular degeneration (AMD) is the main cause of visual impairment and blindness in people aged over 65 years in developed countries. Approximately $30 \%$ of adults aged 75 years or older have some signs of maculopathy and $6 \%$ to $8 \%$ of these individuals suffer significant loss of vision due to advanced stages of AMD. ${ }^{1-4}$

The development of new blood vessels from preexisting vasculature, known as angiogenesis, has a causal role in neovascular AMD. ${ }^{5}$ Vascular endothelial growth factor (VEGF) is a positive regulator of angiogenesis ${ }^{5}$ and its proven role in the pathological neovascularization in wet AMD has provided evidence for the use of anti-VEGF agents as potential therapies. ${ }^{5-8}$

VEGF inhibitors have been established as the mainstay of treatment in wet AMD. Clinical trials have established the efficacy of ranibizumab for the treatment of neovascular AMD. The results of the Minimally Classic/Occult Trial of the Anti-VEGF Antibody Ranibizumab in the Treatment of Neovascular AMD (MARINA) and AntiVEGF Antibody for the Treatment of Predominantly Classic Choroidal Neovascularization in AMD (ANCHOR), study revealed that $95 \%$ of ranibizumab-treated patients maintained visual acuity compared to $62 \%$ of sham or photodynamic therapy (PDT) groups $(P<0.01)$ at 1 year. Furthermore, up to $40 \%$ demonstrated an improvement in vision of at least three lines. ${ }^{9,10}$ In addition, bevacizumab is used off-label to treat AMD, despite the absence of similar supporting data.

However, in spite of ongoing therapies with the current standard anti-VEGF approach, it is unknown why more than half of patients do not improve after anti-VEGF therapy and about $10 \%$ of patients do not respond at all to treatment. Tachyphylaxis or loss of drug effectiveness after administration of bevacizumab and ranibizumab was recently recognized. Resistance to antiangiogenic therapy due to genetic factors appears as another important parameter that cannot be overlooked. 
In this study, we review the literature for the possible causes of failure after treatment with anti-VEGF agents, and attempt to propose an algorithm of suggestive actions to increase the chances of successful management of such difficult cases.

We conducted a comprehensive search of the literature published until 2012 to identify studies evaluating the effects of anti-VEGF therapeutic agents in the management of AMD. These included randomized controlled studies, prospective and retrospective case-control studies, and case series. Studies with other clinical subtypes of AMD were not excluded. English and non-English language articles with abstracts translated into English were retrieved using a keyword search of PubMed/Medline and the National Institutes of Health Clinical Trials databases. Search terms included: 'AMD' or 'age-related macular degeneration', 'RAP' or 'retinal angiomatous proliferation', 'PCV' or 'polypoidal choroidal vasculopathy', 'anti-VEGF + poor response', 'anti-VEGF tachyphylaxis', 'bevacizumab' or 'Avastin', 'ranibizumab' or 'Lucentis', and 'photodynamic therapy'. These searches were supplemented by manually searching the reference lists of all major review articles. A clinical diagnosis of wet AMD was defined by the authors of the trial reports. Clinical studies that met evidence criteria were considered for review.

\section{Anti-VEGF resistance}

Poor response to anti-VEGF may be due to various factors. In nearly $50 \%$ of these 'resistant' cases, patients are misdiagnosed for AMD, while polypoidal choroidal vasculopathy (PCV) accounts for the majority of the underlying pathology. Tachyphylaxis also appears to play a significant role and these patients may require treatment modifications. Finally, a small number of AMD patients may be genetically predisposed to show resistance to anti-VEGF treatment. Considering the systemic risks and cost of therapy involved, identification of these eyes is essential. ${ }^{11}$

The aim of a retrospective study conducted by Manoj between January 2007 and December 2008 was to evaluate eyes with AMD that responded poorly to anti-VEGF therapy and to investigate reasons for treatment failure. ${ }^{11}$ In 24 eyes (46.2\%) of the patients with poor response to treatment, the primary diagnosis of AMD required revision. This group included cases of PCV (19 eyes), retinal angiomatous proliferation (RAP) (four eyes), and vitelliform lesion (one eye). After excluding the misdiagnosed cases, 14 eyes (26.9\%) were identified, which were nonresponders and eleven eyes $(22.2 \%)$ which fulfilled the criteria for diagnosing tachyphylaxis. Six eyes $(11.5 \%)$ developed complications such as retinal pigment epithelium
(RPE) tear, scarring, RPE atrophy, and eventually poor visual outcome.

Since poor response to anti-VEGF treatment is attributed mainly to PCV and RAP is misdiagnosed for wet AMD, these entities will be described in detail.

\section{Anti-VEGF and misdiagnosis Anti-VEGF and PCV}

As mentioned previously, PCV has been suggested as a variant of neovascular AMD. PCV is an exudative maculopathy affecting vision, with clinical features distinct from neovascular AMD. Currently, no evidence-based guidelines exist for its diagnosis and treatment. Combining results from several case series, PCV has a prevalence of $8 \%-13 \%$ among whites and $24 \%-50 \%$ of Asian populations with neovascular AMD. ${ }^{12,13}$ In a retrospective case series, eyes determined as refractory to anti-VEGF therapy in neovascular AMD were found to have PCV and this was thought to be responsible for the lack of treatment response. ${ }^{14}$ Today, the treatment strategies for PCV largely mirror the management of AMD patients, but lack the evidence base of sufficiently controlled clinical trials. Verteporfin PDT has been shown to be the most promising, effective, and safe treatment in symptomatic patients with submacular PCV. ${ }^{15,16}$ However, recurrences and the potential risk of accelerated RPE atrophy highlight the need for additional therapeutic options. ${ }^{17,18}$ Anti-VEGF therapy, which has dramatically changed the management of neovascular AMD, is now more frequently used for PCV patients, but with varying results. Various authors have also suggested that combining PDT with anti-VEGF, may improve the outcome. Furthermore, argon laser photocoagulation has successfully been used to coagulate pure extrafoveal polyps. According to the EVEREST trial, the only published randomized controlled clinical trial in PCV, diagnosis of PCV should be based on early-phase nodular hyperfluorescence from choroidal vasculature visualized using indocyanine green angiography (ICGA). Recommended initial treatment of juxtafoveal and subfoveal PCV is either ICGAguided verteporfin PDT or verteporfin PDT plus $3 \times 0.5 \mathrm{mg}$ ranibizumab intravitreal injections 1 month apart. If there is incomplete regression of polyps by ICGA, eyes should be retreated with verteporfin PDT monotherapy or verteporfin PDT plus ranibizumab. If there is complete regression of polyps by ICGA, but there is leakage on fluorescein angiography and other clinical or anatomical signs of disease activity, eyes should be retreated with ranibizumab. ${ }^{19}$

Thus, it is very important to consider the presence of specific vascular abnormalities, like PCV, in AMD patients who fail to respond to standard anti-VEGF therapy. ${ }^{13}$ The use 
of advanced imaging technologies, specifically ICGA, may help to better identify these cases and to guide alternative treatment strategies such as verteporfin PDT or combined treatments to achieve anatomic restoration and preservation of visual function in these patients.

\section{Anti-VEGF and RAP}

RAP is another recognized entity that responds differently than the typical CNV seen in wet AMD. It has been described as a variant of WET AMD. The term 'RAP' was first introduced by Yannuzzi et al in 2001. ${ }^{20}$ RAP is differentiated into three stages based on clinical and angiographic observations: stage 1 , proliferation of intraretinal capillaries originating from the deep retinal complex (intraretinal neovascularization); stage 2 , growth of the retinal vessels into the subretinal space (subretinal neovascularization); and stage 3, clinically or angiographically observed $\mathrm{CNV}^{20}$

RAP is sometimes referred to as type 3 neovascularization to distinguish it from the type 1 and $2 \mathrm{CNV}$ anatomic classifications described by Freund et al. ${ }^{21}$ It is also described by the term 'retinal anastomosis to the lesion', as it is believed that the CNV lesion precedes the development of an anastomosing retinal vessel to this lesion. ${ }^{22}$

RAP represents $15 \%$ of all neovascular AMD in white patients and $4.5 \%$ of all neovascular AMD in Japanese patients. $^{23,24}$ The natural course of RAP differs from typical wet AMD and has poor visual outcomes. ${ }^{25-27}$ Furthermore, various treatments for RAP such as conventional laser photocoagulation, ${ }^{26,28}$ transpupillary thermotherapy, ${ }^{26,29}$ surgical ablation, ${ }^{30,31}$ and monotherapy with PDT with verteporfin ${ }^{32,33}$ have failed to control the disease adequately. The clinical efficacy may depend on the suppression of CNV using antiVEGF agents. Verteporfin may accumulate minimally in the suppressed neovascular complex after injection of intravitreal anti-VEGF agents. For this reason, PDT may need to be applied as soon as possible after intravitreal bevacizumab. Applying PDT simultaneously with intravitreal anti-VEGF agents may also be effective. ${ }^{34}$ Rouvas et al had reported positive results in all groups of a small prospective series comparing ranibizumab, PDT with intravitreal triamcinolone acetonide (IVT), and PDT with ranibizumab. ${ }^{35}$ Saito et al retrospectively reviewed 13 cases with RAP and reported that combined anti-VEGF and PDT for RAP patients effectively maintained or improved visual acuity (VA) and reduced exudation, without severe adverse events, over 24 months. ${ }^{34}$ In another retrospective study 15 consecutive RAP treatment-naïve eyes were treated with triple therapy of intravitreal bevacizumab injections (IVB), sub-Tenon injec- tion of triamcinolone (ST-TA), and reduced laser fluence PDT (RF-PDT). Whenever there was a recurrence of retinal-retinal anastomosis (RRA) or retinal-choroidal anastomosis (RCA) and marked leakage from subretinal neovascularization, the triple therapy was reapplied. When there were only intraretinal exudative and/or hemorrhagic changes without distinct RRA or RCA, IVB alone was applied. The study concluded that combined IVB, ST-TA, and RF-PDT for RAP was effective in maintaining or improving VA at 1 year. In addition, the number of treatments could be reduced markedly. ${ }^{36}$ Modifications in therapeutic protocols may be indicated to improve visual and anatomic outcomes in populations with RAP. ${ }^{13}$

\section{Anti-VEGF and tachyphylaxis}

Another significant cause of anti-VEGF treatment failure is tachyphylaxis, the diminished therapeutic response to a drug, following repeated administration. ${ }^{37,38}$ Tachyphylaxis to anti-VEGF was defined the lack of treatment response of a reactivated $\mathrm{CNV}$, which had previously been treated successfully with complete resolution of intra/subretinal fluid using the same therapeutic regime. ${ }^{39}$ Inadequate response to anti-VEGF was considered a decrease in vision accompanied by an increase in central retinal thickness (CRT) despite repeated injections. The precise mechanism for the development of tachyphylaxis is unclear. Both local and systemic factors might be involved. It was estimated, that approximately $2 \%$ of the patients may develop tachyphylaxis during their treatment. ${ }^{39}$

The attenuated therapeutic response over time was observed to occur regardless of the initial treatment with ranibizumab or bevacizumab. It was observed that some patients developed a decreased response soon after two antiVEGF injections, while others did not develop tachyphylaxis until they underwent ten or eleven injections. ${ }^{40}$ In the report by Forooghian et al, ${ }^{41}$ the median time to develop tachyphylaxis was 100 weeks, and the median number of intravitreal bevacizumab treatments prior to developing tachyphylaxis was eight. Additionally, Schaal et $\mathrm{al}^{42}$ reported that approximately three injections were required before the efficacy decreased to $50 \%$ of the initial optical coherence tomography response. The improved response was found in patients initially treated with either ranibizumab or bevacizumab. ${ }^{40}$ Patients may respond favorably to a change in the treatment regimen to another anti-VEGF drug. Patients may require multiple injections to demonstrate a favorable treatment response and reversal of the tachyphylaxis effect.

A retrospective review of patients who had received both intravitreal ranibizumab and bevacizumab was performed 
from September 2006 to April 2009 treated for CNV at the Doheny Eye Institute, University of Southern California, Los Angeles, CA, USA. ${ }^{40}$ The aim was to evaluate the effect of switching to bevacizumab or ranibizumab after developing tachyphylaxis during anti-VEGF therapy for CNV. Of 26 eyes, that were included, ten were initially treated with bevacizumab and then subsequently changed to ranibizumab when persistent subretinal fluid, pigment epithelial detachment, and/or central macular edema were observed. Of these, seven had occult CNV and three had predominantly classic CNV. One eye in the occult CNV group did not respond after it had been switched to ranibizumab. Six eyes had a positive therapeutic response, after one injection in four eyes, and after two or three injections in the remaining two eyes. In the classic group, two eyes responded to ranibizumab and one did not. Sixteen eyes were treated with ranibizumab initially before changing to bevacizumab. Of these, 15 had occult CNV and one predominantly classic. Three of the 16 eyes failed to respond to bevacizumab, six improved after one injection, and five after two injections. ${ }^{40}$ The authors concluded that the majority of cases $(81 \%)$ with confirmed tachyphylaxis demonstrated at least some positive response after switching therapies. ${ }^{40}$

\section{Anti-VEGF and genetic factors}

Genetic predisposition may contribute to the variability in response to anti-VEGF treatment. Genetic factors which influence the development of AMD have been primarily identified through association studies with DNA sequence variants. $^{43}$

On the other hand, there are few studies that try to investigate the relationship between genetic factors and response to anti-VEGF therapy. ${ }^{44-46}$

In a recent systematic meta-analysis published by Chen et $\mathrm{al}^{47}$ it is discussed the association between polymorphism rs1061170 in the CFH (Complement Factor H) gene and response to anti-VEGF treatment. The results of 10 studies (1,510 patients), showed that polymorphism rs1061170 was a predictor of treatment response to anti-VEGF agents of neovascular AMD. Additionally, according to that publication, patients homozygous for the variant risk $\mathrm{C}$-allele (CC genotype) corresponded to a decreased response to treatment by approximately 1.6-fold when compared with patients carrying homozygous for the ancestral T-allele (TT genotype). That was probably the first meta-analysis demonstrating that a genetic marker was confirmed to be predictive for AMD treatment response.

In another study, Teper et $\mathrm{al}^{48}$ included 90 patients ( 90 eyes) with exudative AMD treated with ranibizumab and showed that VA did not improve in patients homozygous for the gene
ARMS2 69S, despite a decrease in CRT. The role of ARMS2 has not been fully elucidated; however, some findings suggest that it is involved in the extracellular matrix. ${ }^{37}$

Recently, Orlin et al failed to prove any association between known AMD genetic risk variants in the $\mathrm{CFH}$, ARMS2, and HTRA1 genes and response to anti-VEGF treatment for wet AMD. In their patient cohort, there was no statistically significant association between response to antiVEGF therapy and the genotype in both positive-responder and negative-responder groups. ${ }^{49}$

Overall, single genotypes are likely to explain only a small proportion of efficacy variation. Large samples and genome-wide analyses rather than a candidate gene approach might improve the replication of genetic associations leading to the generation of multivariate predictive models and personalized therapy.

\section{Conclusion}

In conclusion, poor response to anti-VEGF may be associated with misdiagnosis, tachyphylaxis, and genetic factors. Misdiagnosis appears to be a common cause of treatment failure. PCV and RAP may be variants of neovascular AMD and may need combined therapy techniques in order to achieve resolution. For such cases PDT, argon laser or even IVT have been successfully used either as monotherapy or combined with anti-VEGF agents. ${ }^{13}$ The mechanisms of treatment resistance in these cases are poorly understood. The location of the vascular abnormality (inner choroidal circulation in PCV), or the degree of vascular maturity has been suggested as being the main cause of anti-VEGF monotherapy failure in such cases.

Tachyphylaxis, the loss of drug effectiveness over time, may be due to metabolic or cellular factors. Metabolic tolerance is the result of alterations in drug absorption, distribution or metabolism, which decreases the effective concentration of the drug. With cellular tolerance, the response to the drug is decreased by cellular mechanisms. For instance, there could be a reduction in the number or the sensitivity of drug receptors. Moreover, systemic circulating antibodies to bevacizumab and ranibizumab may neutralize the drug's effect. Forooghian et al have speculated that the formation of circulating neutralizing antibodies (NAbs) against bevacizumab may be the cause. ${ }^{41}$ Systemic NAbs against humanized molecules, including ranibizumab, have also been documented by Rosenfeld et al. ${ }^{37}$ If tachyphylaxis is the reason of treatment failure, switching a drug to another (bevacizumab to ranibizumab and vice versa) could be a rational strategy. ${ }^{40}$ According to published data, switching drugs has usually positive effects. Other strategies, as increasing the drug dose, 
or reducing the interval between treatments do not seem to be effective. Nevertheless, in a recent report, the authors have found a pharmacokinetic rationale for dosing every 2 weeks versus 4 weeks with intravitreal ranibizumab, bevacizumab and the newest VEGF trap, aflibercept, in a declining order. The authors suggested that the cases likely to benefit the most by such an intensive treatment are those that have initially responded well to anti-VEGF treatment, but the subretinal fluid recurred earlier than 4 weeks. ${ }^{48}$

Growing evidence in the literature suggests that genetics seems to play a major role in the response to anti-VEGF therapy. Published papers report conflicting results concerning the relationship between genes and drug efficacy. That is partially because the genetic contribution to the variable outcomes in wet AMD treatment appears to be related to many loci. Moreover, single genotypes are likely to explain only a small proportion of efficacy variation. It is also possible that some genotypes predispose to the development of late stages of AMD, but it is not fully understood how these late stages progress or respond to treatment. ${ }^{50}$

Finally, vitreomacular adhesion (VMA) may play a role in the treatment of AMD with anti-VEGFs. A recent paper published by Lee and $\mathrm{Koh}^{51}$ has shown that posterior VMA is associated with an inferior visual outcome after antiVEGF treatment for AMD and vitreomacular traction could antagonize the effect of anti-VEGF treatment; this should be elucidated with future studies.

In summary, anti-VEGF treatment remains the standard treatment for wet AMD although resistance to it is also a fact in a significant amount of cases. The following algorithm is proposed to help in such difficult cases:

1. If anti-VEGF therapy fails from the beginning, misdiagnosis may be the possible explanation. The use of advanced imaging technologies, specifically ICGA, may help to better identify the correct diagnosis, and to guide alternative treatment strategies. If PCV is found to be the case, then either argon laser photocoagulation (if the polyps are extrafoveal), or combined treatments such as PDT and anti-VEGF (if the polyps are subfoveal), could be tried. On the other hand, if RAP is found to be involved, then combined treatment (PDT or IVTA with anti-VEGF) can be used in order to achieve anatomic restoration and preservation of visual function in these patients.

2. If poor response to anti-VEGF develops after an initial successful treatment period, one should think of tachyphylaxis. In these cases, switching the drug from one type of anti-VEGF to the other could be the first action to be attempted. If this will not prove to be successful, trying combined therapies (PDT or triamcinolone with antiVEGF) may be a therapeutic option.

3. If neither of the above is identified, then genetic predisposition or other unknown factors could be responsible for drug resistance. In such cases, switching to another anti-VEGF may be tried, although there is minimal evidence in the literature to support it.

\section{Disclosure}

The authors report no conflicts of interest in this work. The authors alone are responsible for the content and writing of the paper.

\section{References}

1. Kahn HA, Leibowitz HM, Ganley JP, et al. The Framingham Eye Study. I. Outline and major prevalence findings. Am J Epidemiol. 1977;106: 17-32.

2. Klein R, Klein BE, Linton KL. Prevalence of age-related maculopathy. The Beaver Dam Eye Study. Ophthalmology. 1992;99:933-943.

3. Klein BE, Klein R. Cataracts and macular degeneration in older Americans. Arch Ophthalmol. 1982;100:571-573.

4. Mitchell P, Smith W, Attebo K, Wang JJ. Prevalence of agerelated maculopathy in Australia. The Blue Mountains Eye Study. Ophthalmology. 1995;102:1450-1460.

5. Ferrara N, Gerber HP, Lecouter J. The biology of VEGF and its receptors. Nat Med. 2003;9:669-676.

6. Fine SL, Berger JW, Maguire MG, Ho AC. Age-related macular degeneration. $N$ Engl J Med. 2000;342:483-492.

7. Aiello LP, Avery RL, Arrigg PG, et al. Vascular endothelial growth factor in ocular fluid of patients with diabetic retinopathy and other retinal disorders. N Engl J Med. 1994;331:1480-1487.

8. Adamis AP, Shima DT, Tolentino MJ, et al. Inhibition of vascular endothelial growth factor prevents retinal ischemia-associated iris neovascularization in a nonhuman primate. Arch Ophthalmol. 1996;114: 66-71.

9. Presta LG, Chen H, O'Connor SJ, et al. Humanization of an anti-vascular endothelial growth factor monoclonal antibody for the therapy of solid tumors and other disorders. Cancer Res. 1997;57:4593-4599.

10. Hurwitz H, Fehrenbacher L, Novotny W, et al. Bevacizumab plus irinotecan, fluorouracil, and leucovorin for metastatic colorectal cancer. N Engl J Med. 2004;350:2335-2342.

11. Manoj SM. Why does anti VEGF treatment fail in age related macular degeneration (AMD). Kerala Journal of Ophthalmology. 2011.

12. Sho K, Takahashi K, Yamada H, et al. Polypoidal choroidal vasculopathy: incidence, demographic features, and clinical characteristics. Arch Ophthalmol. 2003;121:1392-1396.

13. Cho M, Barbazetto IA, Freund KB. Refractory neovascular age-related macular degeneration secondary to polypoidal choroidal vasculopathy. Am J Ophthalmol. 2009;148:70-78. e1.

14. Seddon JM, Francis PJ, George S, Schultz DW, Rosner B, Klein ML. Association of CFH Y402H and LOC387715 A69S with progression of age-related macular degeneration. JAMA. 2007;297: 1793-1800.

15. Chan WM, Lam DS, Lai TY, et al. Photodynamic therapy with verteporfin for symptomatic polypoidal choroidal vasculopathy: one-year results of a prospective case series. Ophthalmology. 2004;111:1576-1584.

16. Eandi CM, Ober MD, Freund KB, Slakter JS, Yannuzzi LA. Selective photodynamic therapy for neovascular age-related macular degeneration with polypoidal choroidal neovascularization. Retina. 2007;27:825-831. 
17. Wakabayashi T, Gomi F, Sawa M, Tsujikawa M, Tano Y. Marked vascular changes of polypoidal choroidal vasculopathy after photodynamic therapy. Br J Ophthalmol. 2008;92:936-940.

18. Gomi F, Sawa M, Sakaguchi H, et al. Efficacy of intravitreal bevacizumab for polypoidal choroidal vasculopathy. Br J Ophthalmol. 2008;92:70-73.

19. Koh AH, Chen LJ, Chen SJ, et al. Polypoidal choroidal vasculopathy: evidence-based guidelines for clinical diagnosis and treatment. Retina. 2013;33:686-716.

20. Yannuzzi LA, Negrao S, Iida T, et al. Retinal angiomatous proliferation in age-related macular degeneration. Retina. 2001;21:416-434.

21. Freund KB, Ho IV, Barbazetto IA, et al. Type 3 neovascularization: the expanded spectrum of retinal angiomatous proliferation. Retina. 2008;28:201-211.

22. Scott AW, Bressler SB. Retinal angiomatous proliferation or retinal anastomosis to the lesion. Eye (Lond). 2010;24:491-496.

23. Cohen SY, Creuzot-Garcher C, Darmon J, et al. Types of choroidal neovascularisation in newly diagnosed exudative age-related macular degeneration. Br J Ophthalmol. 2007;91:1173-1176.

24. Maruko I, Iida T, Saito M, Nagayama D, Saito K. Clinical characteristics of exudative age-related macular degeneration in Japanese patients. Am J Ophthalmol. 2007;144:15-22.

25. Hartnett ME, Weiter JJ, Staurenghi G, Elsner AE. Deep retinal vascular anomalous complexes in advanced age-related macular degeneration. Ophthalmology. 1996;103:2042-2053.

26. Bottoni F, Massacesi A, Cigada M, Viola F, Musicco I, Staurenghi G. Treatment of retinal angiomatous proliferation in age-related macular degeneration: a series of 104 cases of retinal angiomatous proliferation. Arch Ophthalmol. 2005;123:1644-1650.

27. Bressler NM. Retinal anastomosis to choroidal neovascularization: a bum rap for a difficult disease. Arch Ophthalmol. 2005;123:1741-1743.

28. Slakter JS, Yannuzzi LA, Schneider U, et al. Retinal choroidal anastomoses and occult choroidal neovascularization in age-related macular degeneration. Ophthalmology. 2000;107:742-753; discussion, 753-754.

29. Kuroiwa US, Arai J, Gaun S, Iida T, Yoshimura N. Rapidly progressive scar formation after transpupillary thermotherapy in retinal angiomatous proliferation. Retina. 2003;23:417-420.

30. Sakimoto AS, Gomi F, Sakaguchi H, Tano Y. Recurrent retinal angiomatous proliferation after surgical ablation. Am J Ophthalmol. 2005;139:917-918.

31. Shiragami C, Iida T, Nagayama D, Baba T, Shiraga F. Recurrence after surgical ablation for retinal angiomatous proliferation. Retina. 2007;27:198-203

32. Boscia F, Furino C, Sborgia L, Reibaldi M, Sborgia C. Photodynamic therapy for retinal angiomatous proliferations and pigment epithelium detachment. Am J Ophthalmol. 2004;138:1077-1079.

33. Silva RM, Cachulo ML, Figueira J, De Abreu JR, Cunha-Vaz JG. Chorioretinal anastomosis and photodynamic therapy: a two-year followup study. Graefes Arch Clin Exp Ophthalmol. 2007;245:1131-1139.

34. Saito M, Shiragami C, Shiraga F, Nagayama D, Iida T. Combined intravitreal bevacizumab and photodynamic therapy for retinal angiomatous proliferation. Am J Ophthalmol. 2008;146:935-941. e1.

35. Rouvas AA, Papakostas TD, Ntouraki A, Douvali M, Vergados I, Ladas ID. Photodynamic therapy, ranibizumab, and ranibizumab with photodynamic therapy for the treatment of polypoidal choroidal vasculopathy. Retina. 2011;31:464-474.

Drug Design, Development and Therapy

\section{Publish your work in this journal}

Drug Design, Development and Therapy is an international, peerreviewed open-access journal that spans the spectrum of drug design and development through to clinical applications. Clinical outcomes, patient safety, and programs for the development and effective, safe, and sustained use of medicines are a feature of the journal, which
36. Shirakata Y, Shiragami Y, Shiragami C, et al. One-year results of bevacizumab intravitreal and posterior sub-Tenon injection of triamcinolone acetonide with reduced laser fluence photodynamic therapy for retinal angiomatous proliferation. Jpn J Ophthalmol. 2012;56:599-607.

37. Rosenfeld PJ, Brown DM, Heier JS, et al. Ranibizumab for neovascular age-related macular degeneration. $N$ Engl J Med. 2006;355: 1419-1431.

38. Keane PA, Liakopoulos S, Ongchin SC, et al. Quantitative subanalysis of optical coherence tomography after treatment with ranibizumab for neovascular age-related macular degeneration. Invest Ophthalmol Vis Sci. 2008;49:3115-3120.

39. Eghoj MS, Sorensen TL. Tachyphylaxis during treatment of exudative age-related macular degeneration with ranibizumab. Br J Ophthalmol. 2012;96:21-23.

40. Gasperini JL, Fawzi AA, Khondkaryan A, et al. Bevacizumab and ranibizumab tachyphylaxis in the treatment of choroidal neovascularisation. Br J Ophthalmol. 2012;96:14-20.

41. Forooghian F, Chew EY, Meyerle CB, Cukras C, Wong WT. Investigation of the role of neutralizing antibodies against bevacizumab as mediators of tachyphylaxis. Acta Ophthalmol. 2011;89:e206-e207.

42. Schaal S, Kaplan HJ, Tezel TH. Is there tachyphylaxis to intravitreal anti-vascular endothelial growth factor pharmacotherapy in age-related macular degeneration. Ophthalmology. 2008;115:2199-2205.

43. Swaroop A, Chew EY, Rickman CB, Abecasis GR. Unraveling a multifactorial late-onset disease: from genetic susceptibility to disease mechanisms for age-related macular degeneration. Аппи Rev Genomics Hum Genet. 2009;10:19-43.

44. Feng X, Xiao J, Longville B, et al. Complement factor H Y402H and C-reactive protein polymorphism and photodynamic therapy response in age-related macular degeneration. Ophthalmology. 2009;116: 1908-1912. e1901.

45. Brantley MA, Fang AM, King JM, Tewari A, Kymes SM, Shiels A. Association of complement factor $\mathrm{H}$ and LOC387715 genotypes with response of exudative age-related macular degeneration to intravitreal bevacizumab. Ophthalmology. 2007;93:610-613.

46. Lee AY, Raya AK, Kymes SM, Shiels A, Brantley MA. Pharmacogenetics of complement factor $\mathrm{H}(\mathrm{Y} 402 \mathrm{H})$ and treatment of exudative age-related macular degeneration with ranibizumab. Br J Ophthalmology. 2009;93: 610-613.

47. Chen $\mathrm{H}, \mathrm{Yu} \mathrm{KD}, \mathrm{Xu}$ GZ. Association between variant $\mathrm{Y} 402 \mathrm{H}$ in agerelated macular degeneration (AMD) susceptibility gene CFH and treatment response of AMD: a meta-analysis. PLoS One. 2012;7(8):e42464. doi: 10.1371/journal.pone.0042464. Epub 2012 Aug 14.

48. Teper SJ, Nowinska A, Pilat J, Palucha A, Wylegala E. Involvement of genetic factors in the response to a variable-dosing ranibizumab treatment regimen for age-related macular degeneration. Mol Vis. 2010;16: 2598-2604.

49. Orlin A, Hadley D, Chang W, et al. Association between high-risk disease loci and response to anti-vascular endothelial growth factor treatment for wet age related macular degeneration. Retina. 2012;32:4-9.

50. Stewart MW, Rosenfeld PJ, Penha FM, et al. Pharmacokinetic rationale for dosing every 2 weeks versus 4 weeks with intravitreal ranibizumab, bevacizumab, and aflibercept (vascular endothelial growth factor trapeye). Retina. 2011;32:434-457.

51. Lee SJ, Koh HJ. Effects of vitreomacular adhesion on anti-vascular endothelial growth factor treatment for exudative age-related macular degeneration. Ophthalmology. 2011;118:101-110.

\section{Dovepress}

has also been accepted for indexing on PubMed Central. The manuscript management system is completely online and includes a very quick and fair peer-review system, which is all easy to use. Visit http://www.dovepress.com/testimonials.php to read real quotes from published authors.

Submit your manuscript here: http://www.dovepress.com/drug-design-development-and-therapy-journal 